\title{
Chest CT Imaging Spectrum of Coronavirus Disease 2019 (COVID-19) Pneumonia: A Pictorial Essay
}

\author{
Mehdi Khazaei ${ }^{1}$, Arash Mahdavi (iD) ${ }^{1}$, Zahra Mahboubi-Fooladi ${ }^{1}$, Yashar Moharamzad ${ }^{1}$, Reyhaneh \\ Asgari ${ }^{1}$, Ehsan Zarei ${ }^{1}$ and Morteza Sanei Taheri (iD ${ }^{1, *}$ \\ ${ }^{1}$ Department of Radiology, Shahid Beheshti University of Medical Sciences, Tehran, Iran \\ "Corresponding author: Department of Radiology, Shahid Beheshti University of Medical Sciences, Tehran, Iran. Email: saneim@gmail.com
}

Received 2020 April 01; Revised 2020 April 19; Accepted 2020 May 02.

\begin{abstract}
Various manifestations may appear in chest computed tomography (CT) scan of patients with coronavirus disease 2019 (COVID-19). In this pictorial review, we present chest CT manifestations of 14 patients with laboratory-confirmed COVID-19 disease and various manifestations including ground-glass and consolidative opacities, reticular opacities, halo sign, and other findings.
\end{abstract}

Keywords: Coronavirus, COVID-19, Chest, Computed Tomography

\section{Context}

Coronavirus disease 2019 (COVID-19), caused by a novel coronavirus, was first reported from Wuhan city, China, in December 2019 and rapidly spread across the world $(1,2)$. The virus was named severe acute respiratory syndrome coronavirus 2 (SARS-CoV-2) (3). Pneumonia is the most serious manifestation of COVID-19 that mainly affects the respiratory system (1). As the infection evolves, various computed tomography (CT) scan manifestations may appear. Being familiar with these diverse CT findings is important as currently the infection has reached a pandemic; especially in centers that laboratory tests may not be easily accessible.

\section{Evidence Acquisition}

A wide spectrum of CT findings has been reported in COVID-19 pneumonia. Abnormal findings on chest CT could be unilateral or bilateral with single or multilobar involvement. Additionally, the lesions can have various dis tribution patterns (central, peripheral or diffuse) (2). In this pictorial review, we present various manifestations of COVID-19 pneumonia on chest CT scans of 14 patients with positive polymerase-chain reaction (PCR) assay for COVID19. Wherever possible, we describe the CT findings using the standard nomenclature presented by the Fleischner Society as glossary of terms for thoracic imaging (4). The common radiologic findings on chest CT scan of patients with PCR-positive assay for COVID-19 are as follow:

\subsection{Ground Glass Opacity and Consolidation}

Most of the studies reported isolated ground glass opacity as the most frequent finding in COVID-19 pneumonia. According to a systematic review (1), bilateral multilobar ground glass opacities with a peripheral or posterior distribution, mainly in the lower lobes are known features of COVID-19 pneumonia on the initial CT scan. Combination of ground glass and consolidative opacities is a less frequent presentation that is mainly reported in older patients (1). Ground glass opacity is presented in cases 1 to 10 , 12 , and 13. Consolidation is presented in cases 6 to 12 and 14 .

\subsection{Air Bronchogram}

Air bronchogram, as defined by Fleischner Society, is a pattern of air-filled bronchi within a surrounding opaque lung. Consolidation that is a type of increased lung attenuation due to alveolar-filling processes could occur with or without air bronchogram (4). It has been described in up to $80 \%$ of patients with COVID-19 pneumonia (5). Air bronchogram is depicted in case number 7 .

\subsection{Vascular Dilation or Thickening}

Vascular dilation occurs in small vessels within or around a lung parenchymal lesion like a ground glass opacity or consolidation. A recently published study reported vascular thickening in $80 \%$ of cases (6), although in other studies it has been reported as a less frequent finding. Vascular dilation is presented in case number 10. 


\subsection{Reticular Pattern}

The reticular pattern as a dominant pattern (including coarse linear or curvilinear opacity or fine sub-pleural reticulation) is a late and rare finding (7). Moreover, the presence of reticular opacities in combination with other findings has been described as a common presentation of COVID-19 pneumonia especially in the late stage (3). Enlarged fibrous stripes refer to the interstitial septal thickening (1). Small number fibrous stripes are seen in early stages of COVID-19 pneumonia. Nevertheless, in advance stages, more and enlarged fibrous stripes are presented (8, 9 ). Reticular opacities are presented in cases 4, 6, 11, and 12 .

\subsection{Crazy Paving}

Crazy paving pattern refers to ground glass opacities associated with thickened intralobular and interlobular septum (4). It is a common pattern in early stages of COVID19 (8). In addition, development of crazy paving pattern could be seen in intermediate stages of the infection (1). Crazy paving pattern is depicted in case number 10.

\subsection{Bronchial Wall Thickening and Bronchiectasis}

Bronchial wall thickening and bronchiectasis are seen less commonly in COVID-19 pneumonia and are more frequent in later stages (2). Bronchiectasis is presented in case number 2 and bronchial wall thickening is seen in case number 14

\subsection{Halo Sign}

The halo sign is the ground-glass opacity surrounding a mass or nodule (4). Han et al. (6) reported the halo sign in about $65 \%$ of cases, but other studies reported this sign as a rare finding in COVID-19 pneumonia (1). Halo sign is presented in case 7.

\subsection{Reversed Halo Sign}

The reversed halo sign is defined as a central focal area of ground glass opacity surrounded by a complete or incomplete ring of consolidation (4). It has been more reported in later stages of COVID-19 pneumonia (10). The reversed halo sign is presented in case number 8 .

\subsection{Rare Findings}

Mediastinal and hilar lymph node enlargement, discrete/centrilobular pulmonary nodules, pleural thickening, and pleural and pericardial effusion are uncommon findings in CT scan of patients with COVID-19 pneumonia $(11,12)$. Pleural thickening is presented in case number 13. Pleural effusion is noted in case number 11 .

\section{Results}

\subsection{Patient 1}

COVID-19 pneumonia was diagnosed in a 34-year-old man who presented about 48 hours after the onset of fever, non-productive cough, dyspnea and myalgia. Blood oxygen saturation on admission was $94 \%$. Unenhanced CT scan of the chest at the time of admission demonstrated multiple peripheral patchy ground glass opacities with bilateral distribution (Figure 1).

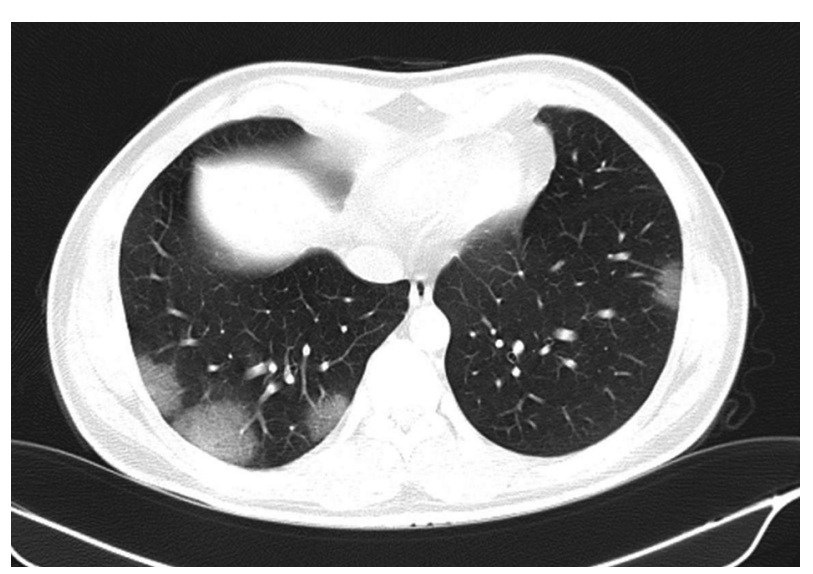

Figure 1. Multiple peripheral patchy ground-glass opacities with bilateral distribution

\subsection{Patient 2}

The patient was a 65-year-old woman with COVID-19 pneumonia who presented with fever and cough. Blood oxygen saturation on admission was $93 \%$. CT scan obtained on day 4 of admission showed bilateral peripheral and subpleural consolidative opacities mainly in the lower lobes. Scattered bronchiectasis was noted (Figure 2).

\subsection{Patient 3}

The patient was a 72-year-old female with COVID-19 pneumonia who presented with fever and shortness of breath. Blood oxygen saturation on admission was $78 \%$. Unenhanced CT scan of the chest indicated extensive ground glass opacities in both lungs (Figure 3 ).

\subsection{Patient 4}

This is a COVID-19 pneumonia in a 36-year-old female who presented with dyspnea and flu-like symptoms. Her initial oxygen saturation was 95\% under ambient air. Spiral CT scan of the thorax revealed patchy sub-pleural ground glass and reticular opacities in both lower lobes (Figure 4). Moreover, situs inversus totalis was noted. The patient did not have any history of recurrent pulmonary or sinonasal infection suggestive of primary ciliary dyskinesia. 


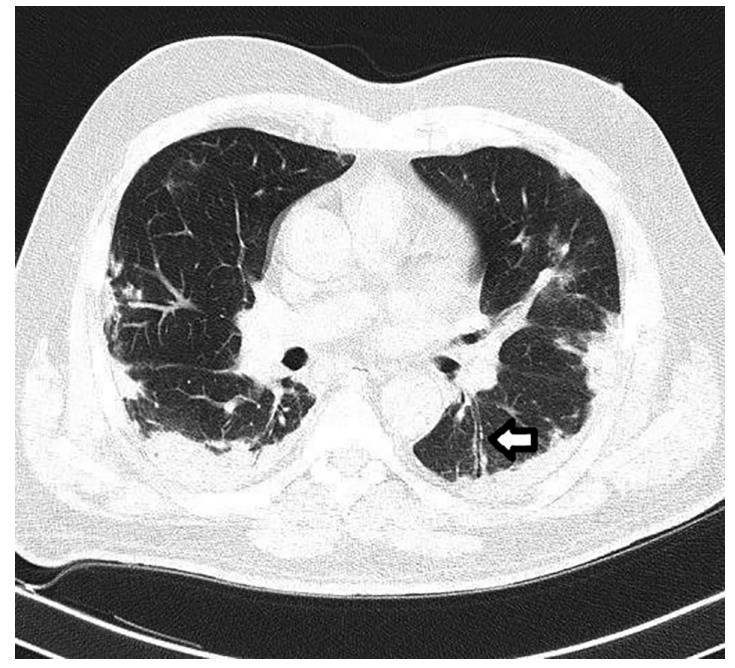

Figure 2. Bilateral peripheral and sub-pleural consolidative opacities mainly in the lower lobes. Scattered bronchiectasis is noted. The arrow shows an ectatic bronchus.

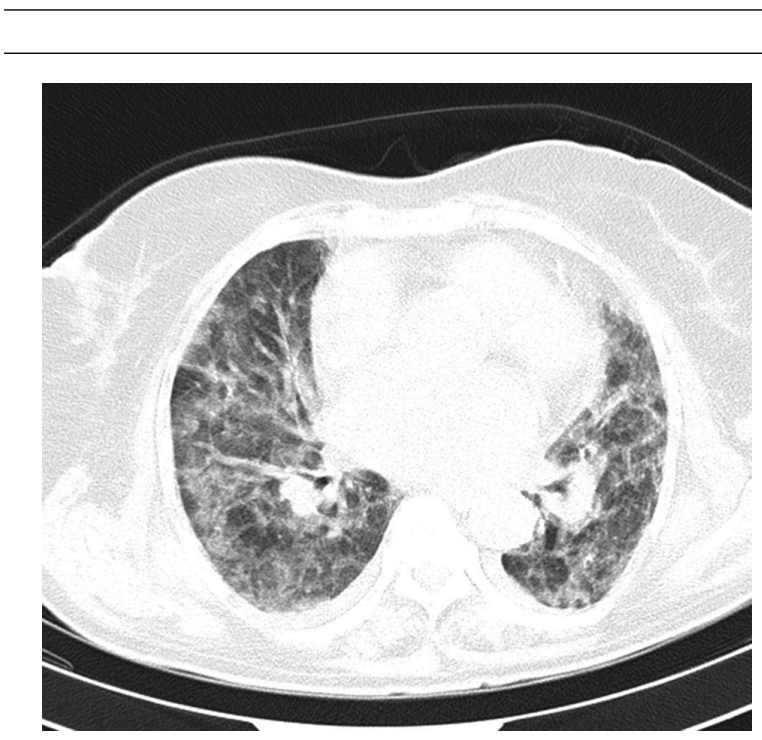

Figure 3. Extensive ground-glass opacities in both lungs

\subsection{Patient 5}

The patient was a 36-year-old female who presented with cough and low-grade fever. Spiral CT scan of the chest at the time of admission demonstrated solitary peripheral ground glass opacity in the posterior segment of the right lower lobe (Figure 5).

\subsection{Patient 6}

The patient was a 68-year-old female with diabetes mellitus (DM) and ischemic heart disease (IHD) who presented with cough, fever, and chills. Blood oxygen saturation on admission was $94 \%$. Spiral CT scan of the chest at the time

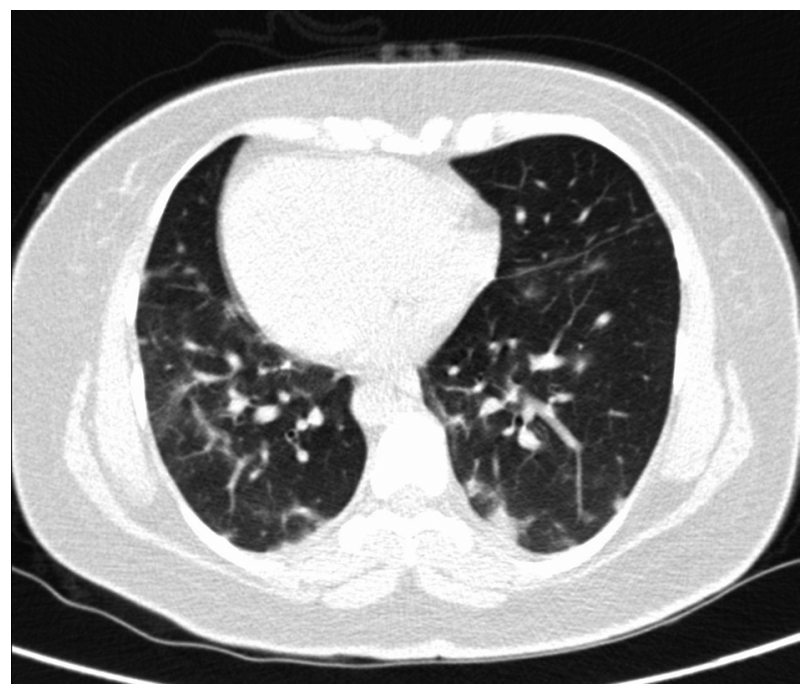

Figure 4. Patchy sub-pleural ground glass and reticular opacities in both lower lobes

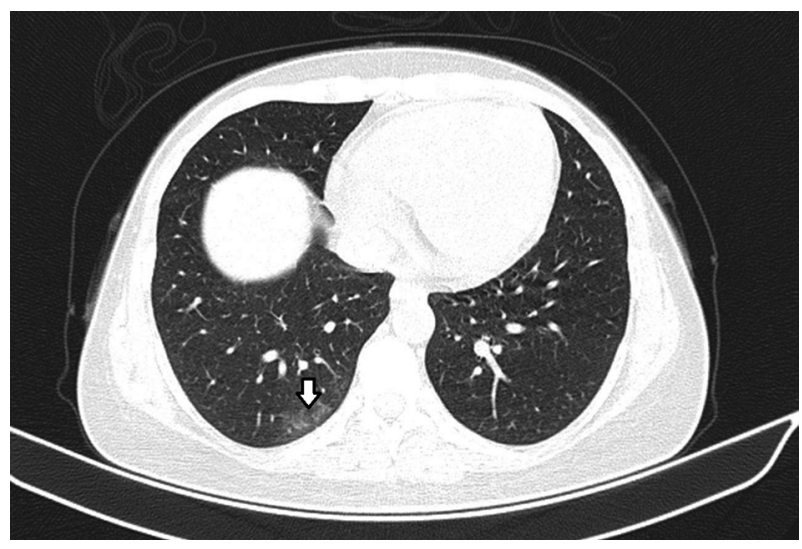

Figure 5. Solitary peripheral ground glass opacity in the posterior segment of the right lower lobe (arrow)

of admission showed bilateral mixed ground glass opacities and consolidation associated with air bronchogram in the upper zones. Reticulation and peripheral linear strip were noted in the basilar segments of both lungs (Figure $6)$.

\subsection{Patient 7}

The patient was a 36-year-old female who presented with non-productive cough and fever. Blood oxygen saturation was $94 \%$ on admission. Spiral CT scan of the chest showed a patch of airspace consolidation with airbronchogram and peripheral ground glass opacity (halo sign) in the right upper lobe (Figure 7). 

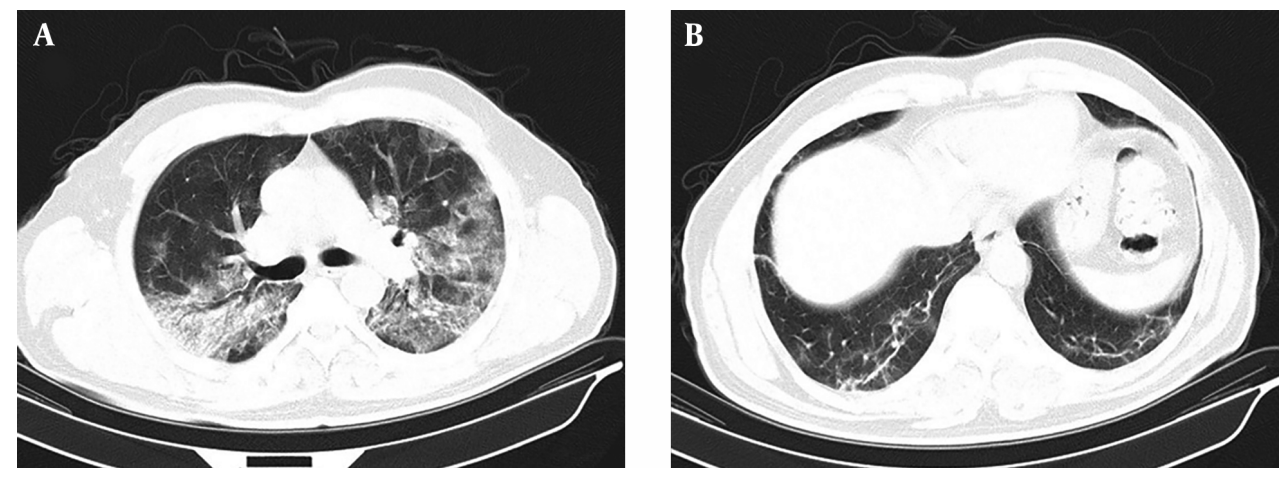

Figure 6. Reticulation and peripheral linear strip are noted in the basilar segments of both lungs

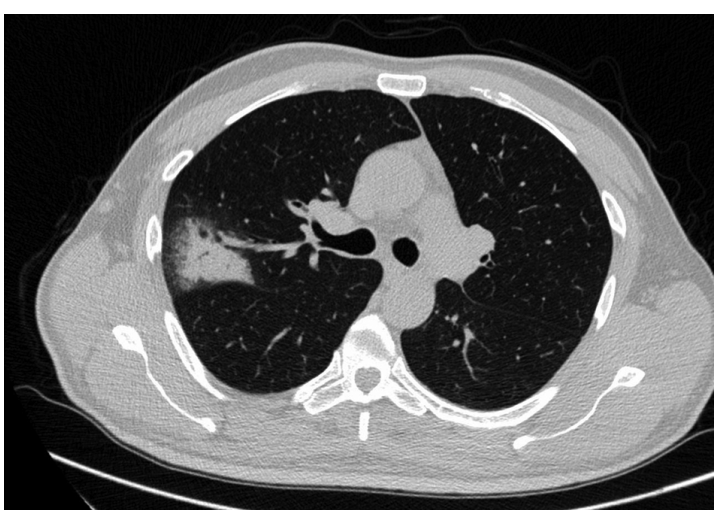

Figure 7. A patch of airspace consolidation with air-bronchogram and peripheral ground glass opacity (halo sign) in the right upper lobe

\subsection{Patient 8}

The patient was a 35-year-old man that complained of fever, cough, shortness of breath and sore throat. His initial blood oxygen saturation was $87 \%$ in room air. Spiral low dose CT scan of the chest revealed patches of ground glass opacities and consolidation mainly in sub-pleural regions of both lungs, some of them representing a reversed halo sign (Figure 8).

\subsection{Patient 9}

The patient was a 66-year-old female with DM, hypertension and chronic kidney disease. She presented with dyspnea and blood oxygen saturation was $92 \%$. Her baseline CT scan of the chest showed peripheral rim of mixed ground glass and consolidative opacities in both lungs that spared the juxta-pleural line (Figure 9).

\subsection{Patient 10}

COVID-19 pneumonia was diagnosed in a 54-year-old man with a 3-day history of cough and fever. Oxygen sat-

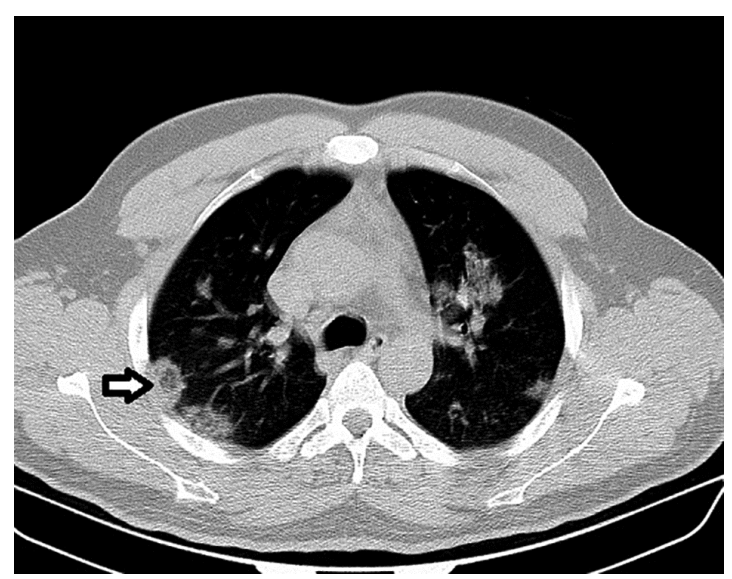

Figure 8. Patches of ground glass opacities and consolidation mainly in sub-pleural regions of both lungs, some of them representing reversed halo sign (arrow)

uration was 93\% under ambient air. CT of the thorax revealed patches of peripherally distributed ground glass opacities as well as consolidation throughout both lungs (Figure 10A). Ground glass opacity in the left upper lobe was associated with thickened intralobular and interlobular septum (crazy paving) (Figure 10B and C). Vascular dilatation was noted within the ground glass opacity of the right upper lobe.

\subsection{Patient 11}

An 80-year-old male presented with dyspnea and fever. Blood oxygen saturation was $94 \%$ in room air. His past medical history was significant for IHD and chronic obstructive pulmonary disease (COPD). Initial spiral low dose CT scan of the thorax revealed patchy air-space consolidation with minimal left sided pleural effusion (Figure 11A). In follow up CT scan that was performed 8 days later, most of the air-space consolidations disappeared. Areas of patchy 


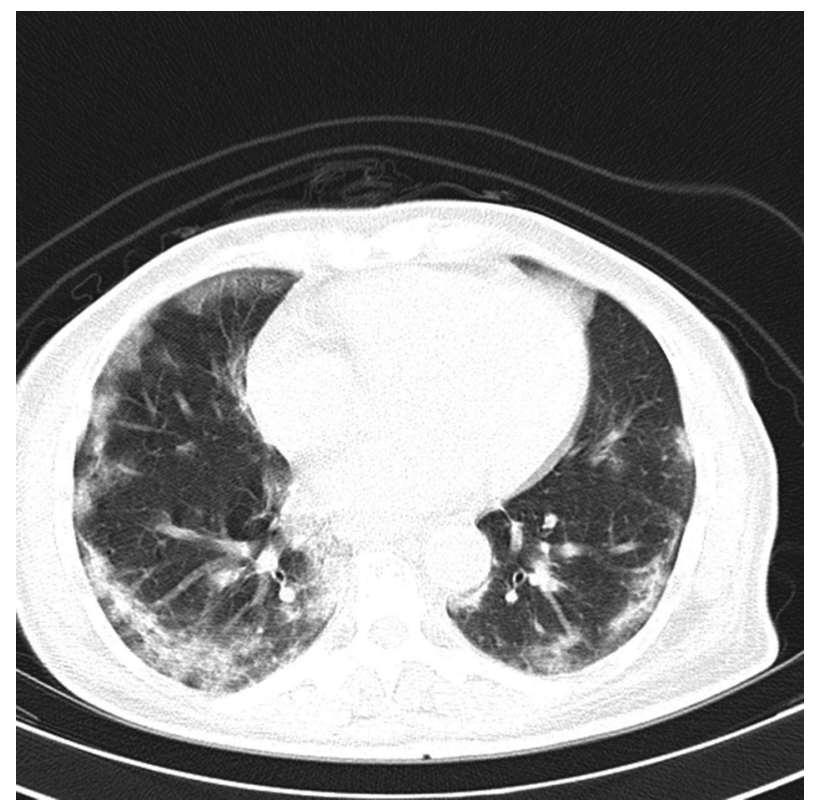

Figure 9. Peripheral rim of mixed ground glass and consolidative opacities in both lungs that spare the juxta-pleural line

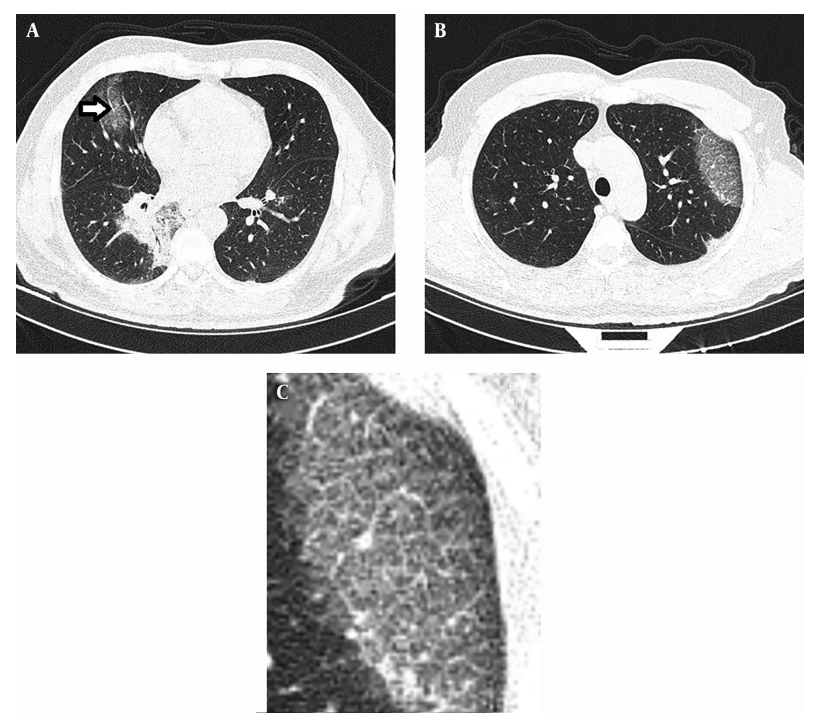

Figure 10. A, Patches of peripherally distributed ground glass opacities as well as consolidation throughout both lungs. Vascular dilatation is noted within ground glass opacity of the right upper lobe (arrow). B and C, Ground glass opacity in the left upper lobe is associated with thickened intralobular and interlobular septum (crazy paving)

reticular and ground glass opacities in sub-pleural regions of both lungs were noted (Figure $11 \mathrm{~B}$ and C).
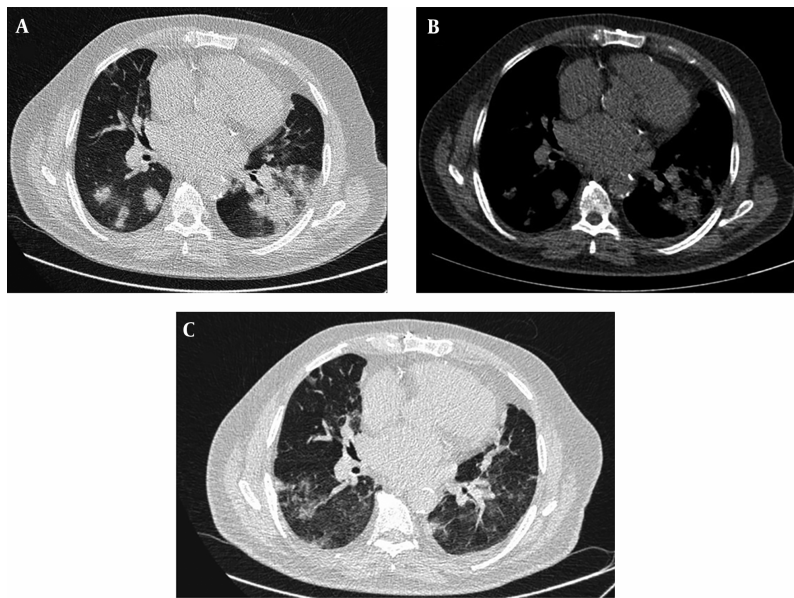

Figure 11. A, Patchy air-space consolidation with minimal left sided pleural effusion $B$ and C, In follow up CT scan that was performed 8 days later, most of the air-space consolidations disappeared. Areas of patchy ground glass and reticular opacities in sub-pleural regions of both lungs are noted.

\subsection{Patient 12}

The patient was a 66-year-old male who presented with fever, dyspnea and oxygen saturation of $88 \%$. Initial spiral low dose CT scan of the thorax revealed diffuse patchy ground glass opacities and consolidation involving most of the both lungs' parenchyma that was compatible with diffuse alveolar damage (Figure 12A). After 8 days, the second CT scan was obtained. Ground glass opacities and consolidations were reduced but increased reticular opacities were noted (Figure 12B).

\subsection{Patient 13}

A 45-year-old man presented about 36 hours after initiation of fever and cough. Initial spiral CT scan of the thorax without contrast did not show any abnormality (Figure 13A). He tested positive for COVID-19 and underwent outpatient therapy. Despite the cessation of fever and reduction of cough and myalgia, the patient returned with pleuritic chest pain located in the right parasternal region 15 days after disease onset. A second CT scan of the thorax was obtained that showed patchy ground glass opacities in both lungs, of which some were located adjacent to the pleura (Figure 13B). Minimal thickening of the pleura was noted.

\subsection{Patient 14}

The patient was a 66-year-old man with a history of seizures. He presented with dyspnea. His oxygen saturation was 92\%. His baseline chest CT scan showed mixed ground glass and consolidative opacities in the left lower lobe (Figure 14A and B). Bronchial wall thickening was noted within and around the lesion. 

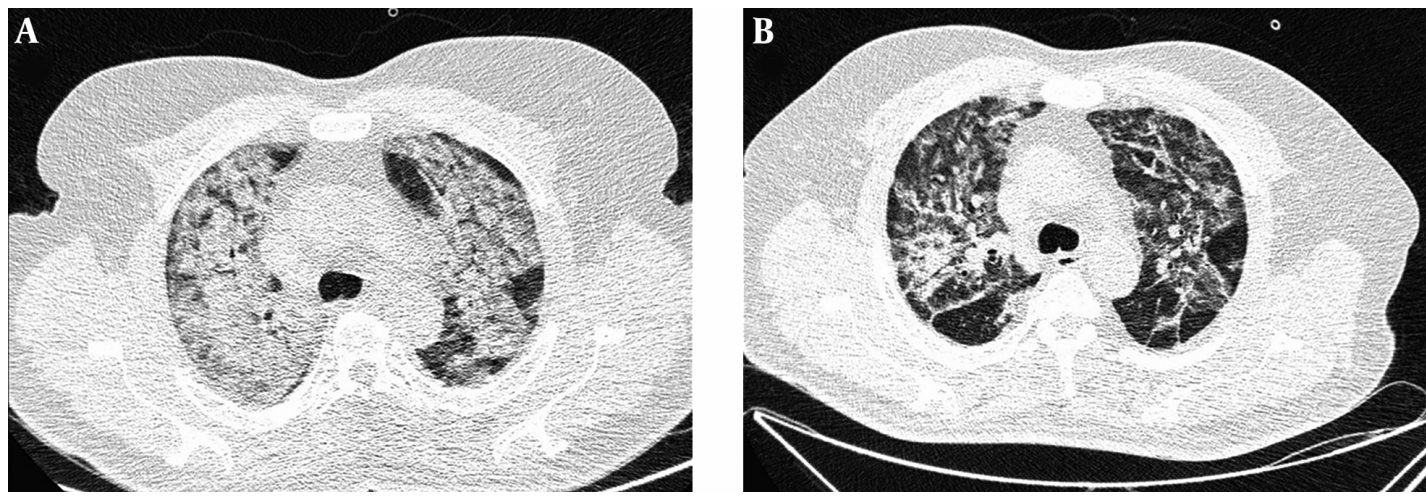

Figure 12. A, Diffuse patchy ground glass opacities and consolidation involving most of the both lungs' parenchyma that is compatible with diffuse alveolar damage. B, After 8 days, a second CT scan was obtained. Ground glass opacities and consolidations were reduced but increased reticular opacities are noted.
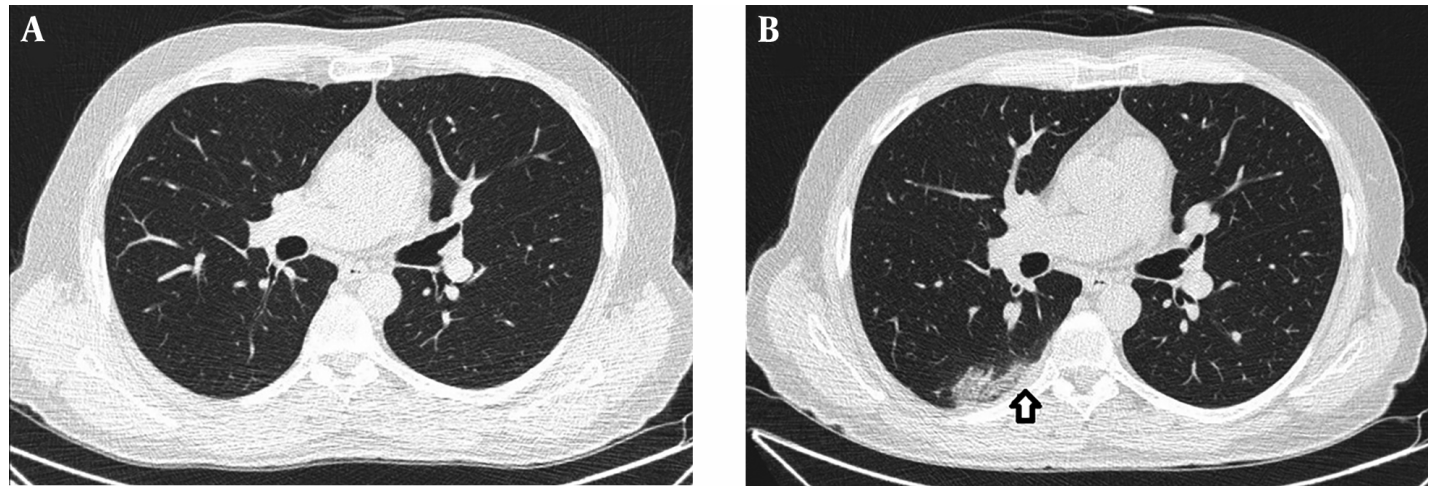

Figure 13. A, Normal initial spiral CT scan of the thorax. B, Second CT scan shows patchy ground glass opacities in both lungs which some of them are located adjacent to the pleura. Minimal thickening of pleura is noted in this image (arrow).
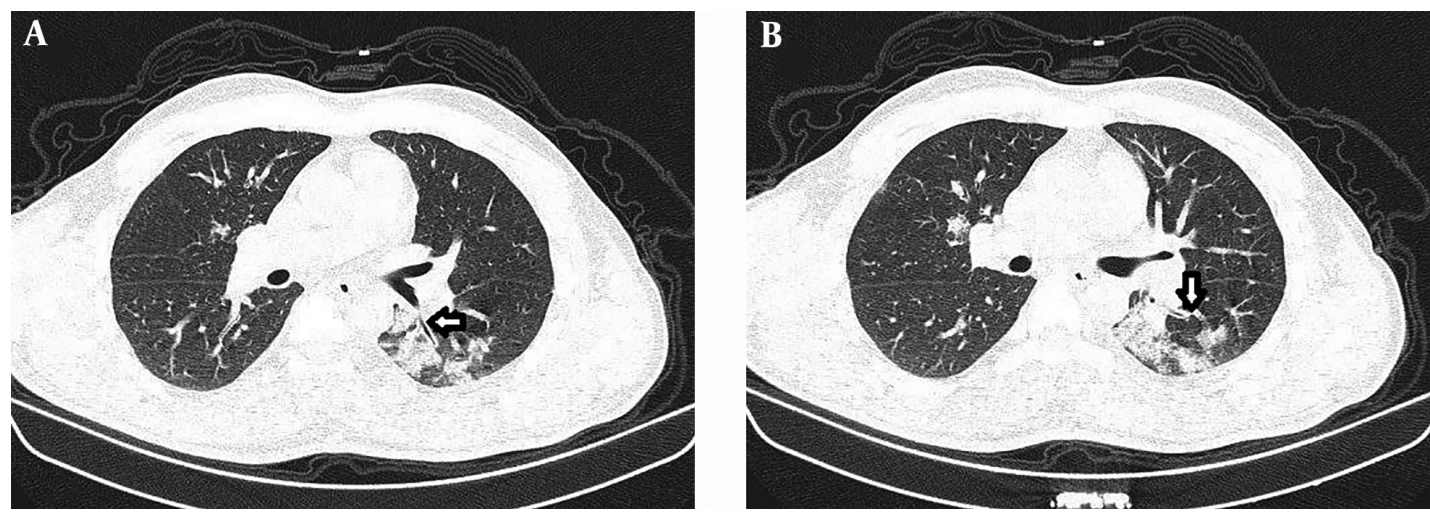

Figure 14. A and B, Mixed ground glass and consolidative opacities in the left lower lobe. Bronchial wall thickening is noted within and around the lesion (arrows).

\section{Conclusion}

As COVID-19 outbreak spreads and many patients who present to the medical centers have respiratory symptoms, chest CT scan is helpful in the management of patients. Radiologists can use the presented cases and images in this pictorial review to improve their skills in the diagnosis of COVID-19 pneumonia. 


\section{Footnotes}

Authors' Contributions: Morteza Sanei Taheri proposed the concept. Mehdi Khazaei, Arash Mahdavi, Zahra Mahboubi-Fooladi, and Morteza Sanei Taheri gathered the data. Yashar Moharamzad reviewed the literature and prepared the draft. Morteza Sanei Taheri, Reyhaneh Asgari, Ehsan Zarei and Mehdi Khazaei revised the manuscript.

Conflict of Interests: No conflict of interest.

Ethical Approval: As this is a pictorial review of the patients for whom chest CT scan was indicated to diagnose the infection (i.e., pneumonia), and the authors reviewed the CT manifestations retrospectively, ethical approval was not required.

Funding/Support: No fund was received.

\section{References}

1. Salehi S, Abedi A, Balakrishnan S, Gholamrezanezhad A. Coronavirus Disease 2019 (COVID-19): A Systematic Review of Imaging Findings in 919 Patients. AJR Am J Roentgenol. 2020:1-7. doi: 10.2214/AJR.20.23034. [PubMed: 32174129].

2. Shi H, Han X, Jiang N, Cao Y, Alwalid O, Gu J, et al. Radiological findings from 81 patients with COVID-19 pneumonia in Wuhan, China: a descriptive study. The Lancet Infectious Diseases. 2020;20(4):425-34. doi: 10.1016/s1473-3099(20)30086-4.

3. Zhou S, Wang Y, Zhu T, Xia L. CT Features of Coronavirus Disease 2019 (COVID-19) Pneumonia in 62 Patients in Wuhan, China. American Journal of Roentgenology. 2020:1-8. doi: 10.2214/ajr.20.22975.
4. Hansell DM, Bankier AA, MacMahon H, McLoud TC, Muller NL, Remy J. Fleischner Society: glossary of terms for thoracic imaging. Radiology. 2008;246(3):697-722. doi: 10.1148/radiol.2462070712. [PubMed: 18195376].

5. Song F, Shi N, Shan F, Zhang Z, Shen J, Lu H, et al. Emerging 2019 Novel Coronavirus (2019-nCoV) Pneumonia. Radiology. 2020;295(1):210-7. doi: 10.1148/radiol.2020200274. [PubMed: 32027573].

6. Han R, Huang L, Jiang H, Dong J, Peng H, Zhang D. Early Clinical and CT Manifestations of Coronavirus Disease 2019 (COVID-19) Pneumonia. AJR Am J Roentgenol. 2020:1-6. doi: 10.2214/AJR.20.22961. [PubMed: 32181672].

7. Wang Y, Dong C, Hu Y, Li C, Ren Q, Zhang X, et al. Temporal Changes of CT Findings in 90 Patients with COVID-19 Pneumonia: A Longitudinal Study. Radiology. 2020:200843. doi: 10.1148/radiol.2020200843. [PubMed: 32191587].

8. Pan Y, Guan H, Zhou S, Wang Y, Li Q, Zhu T, et al. Initial CT findings and temporal changes in patients with the novel coronavirus pneumonia (2019-nCoV): a study of 63 patients in Wuhan, China. European Radiology. 2020. doi: 10.1007/s00330-020-06731-x.

9. Wang J, Liu J, Wang Y, Liu W, Chen X, Sun C, et al. [Dynamic changes of chest CT imaging in patients with corona virus disease-19 (COVID-19)]. Zhejiang Da Xue Xue Bao Yi Xue Ban. 2020;49(1):0. [PubMed: 32096366].

10. Bernheim A, Mei X, Huang M, Yang Y, Fayad ZA, Zhang N, et al. Chest CT Findings in Coronavirus Disease-19 (COVID-19): Relationship to Duration of Infection. Radiology. 2020:200463. doi: 10.1148/radiol.2020200463. [PubMed: 32077789].

11. Kanne JP. Chest CT Findings in 2019 Novel Coronavirus (2019-nCoV) Infections from Wuhan, China: Key Points for the Radiologist. Radiology. 2020;295(1):16-7. doi: 10.1148/radiol.2020200241. [PubMed: 32017662].

12. Jin YH, Cai L, Cheng ZS, Cheng H, Deng T, Fan YP, et al. A rapid advice guideline for the diagnosis and treatment of 2019 novel coronavirus (2019-nCoV) infected pneumonia (standard version). Mil Med Res. 2020;7(1):4. doi: 10.1186/s40779-020-0233-6. [PubMed: 32029004]. [PubMed Central: PMC7003341]. 